\title{
Dynamically Templated Acquisition of EDS X-ray Spectral Images Using Electron Image Contrast
}

\author{
Stephen M. Seddio ${ }^{1}$. \\ 1. Thermo Fisher Scientific, Madison, WI, USA.
}

With modern EDS X-ray microanalysis systems, elemental and phase distributions in a sample are typically determined from hyperspectral images (commonly referred to as EDS "spectral images"), in which a complete EDS spectrum is acquired at every pixel. The resolution of EDS spectral images can match the resolution of electron images acquired by the electron microscope to which the EDS system is attached. Automated routines enable large area mapping, in which these high resolution spectral images can be acquired over entire samples. Such analysis results in the acquisition of millions of pixels, which, in reality, is the acquisition of millions of EDS spectra. Even if EDS detectors are acquiring and storing $\mathrm{X}$-rays at a rate of 1,000,000 counts per second, during the acquisition of a large area map containing 10,000,000 pixels (e.g., a 3,200 × 3,200 pixel map), each pixel would see a count rate of 10 counts per second. The true count rate per pixel would actually be even lower after overhead time costs such as stage movement are considered. Large area acquisitions can require long acquisition times in order to accumulate a statistically meaningful number of counts per pixel. Thus, the trend for EDS systems has been to develop time saving tools to enable rapid data collection (e.g., large active area SDDs) and to enable the extraction of maps with the fewest possible counts per pixel (e.g., multivariate curve resolution $[1,2]$ algorithms such as the Thermo Scientific COMPASS algorithm).

An alternative approach, which can and has been combined with other time saving methods, is for the analyst to restrict a spectral image acquisition only to areas of interest. Many samples contain phases that contain no useful information to the analyst. For example, many samples are embedded in epoxy which may be damaged when exposed the probe doses commonly used for EDS spectral imaging (aside from adding unnecessary time to an acquisition). The phases of interest in metal samples are typically small inclusions or precipitates, which are surrounded by a well-understood alloy. In these cases, the analyst could draw selected areas around the phases of interest for spectral image acquisition by interaction with an electron image, typically a BSE (backscattered electron image). However, such a technique is impractical for automated large area analysis because it would require careful monitoring of the analysis and the analyst's selection of areas for analysis for each spectral image.

Dynamically templated EDS spectral imaging is a recent innovation enabling the automated selection and exclusion areas for acquisition based on contrast thresholding of the electron image that accompanies each EDS spectral image. Areas (or phases) of interest are defined at the beginning of the acquisition by the analyst acquiring an electron image with contrast representative of the phases of interest in the sample and setting thresholds in the histogram of the electron image. After this setup, X-ray data will only be acquired in pixels of spectral images that have electron image contrast that meets the thresholding. In so doing, no $\mathrm{X}$-ray data is acquired for pixels that represent areas of no interest, minimizing the acquisition time.

Fig. 1 was acquired over a portion of a granite sample mounted in epoxy (black, BSE image). Pixels representing epoxy, surface contamination, and fractures had the darkest contrast in the BSE image and were excluded from X-ray acquisition. Fig. 2 was acquired over a granite sample mounted in epoxy. X-ray data was acquired only from pixels that had the brightest contrast in the BSE image which are titanite (red, 
$\mathrm{CaTiSiO}_{5}$ ), zircon (blue, $\mathrm{ZrSiO}_{4}$ ), and a (possibly multiple) rare earth element silicate or carbonate (green).

\section{References:}

[1] Keenan et al., Meth. of Multivariate Spectral Analysis. Pat. 6,675,106 B1. 06Jan.2004.

[2] Keenan et al., Apparatus and Sys. for Multivariate Spectral Analysis. Pat. 6,675,106 B1. 06Jan.2004.

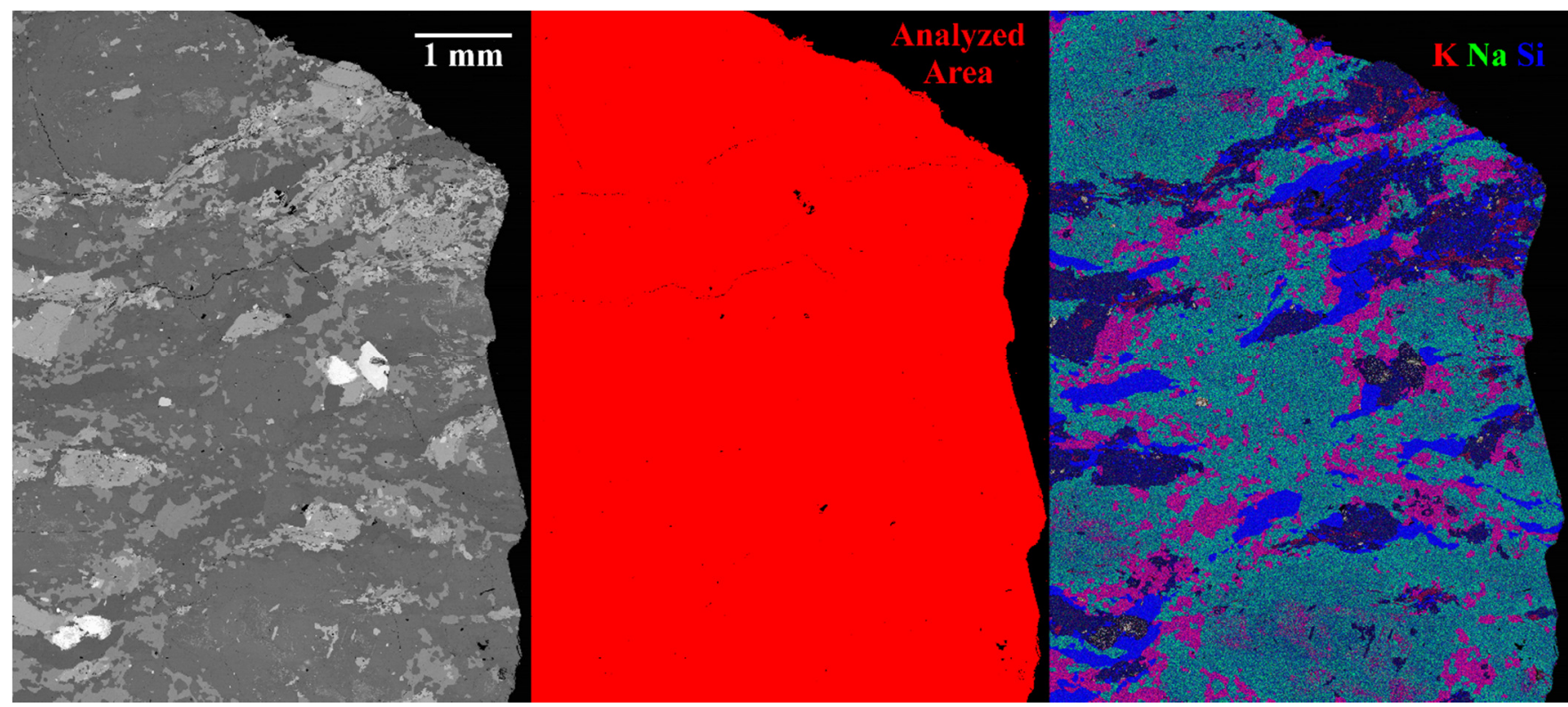

Figure 1. A $9 \times 16$ montage of EDS spectral images $(256 \times 192$ pixels each $)$ acquired from a granite sample mounted in epoxy. Left: BSE image. Center: red pixels indicate pixels with electron contrast that fit the contrast threshold specified for acquisition. Right: RGB composite image of net counts (background removed, peaks deconvolved) elemental X-ray maps of $\mathrm{K}$ (red), $\mathrm{Na}$ (green), and Si (blue).

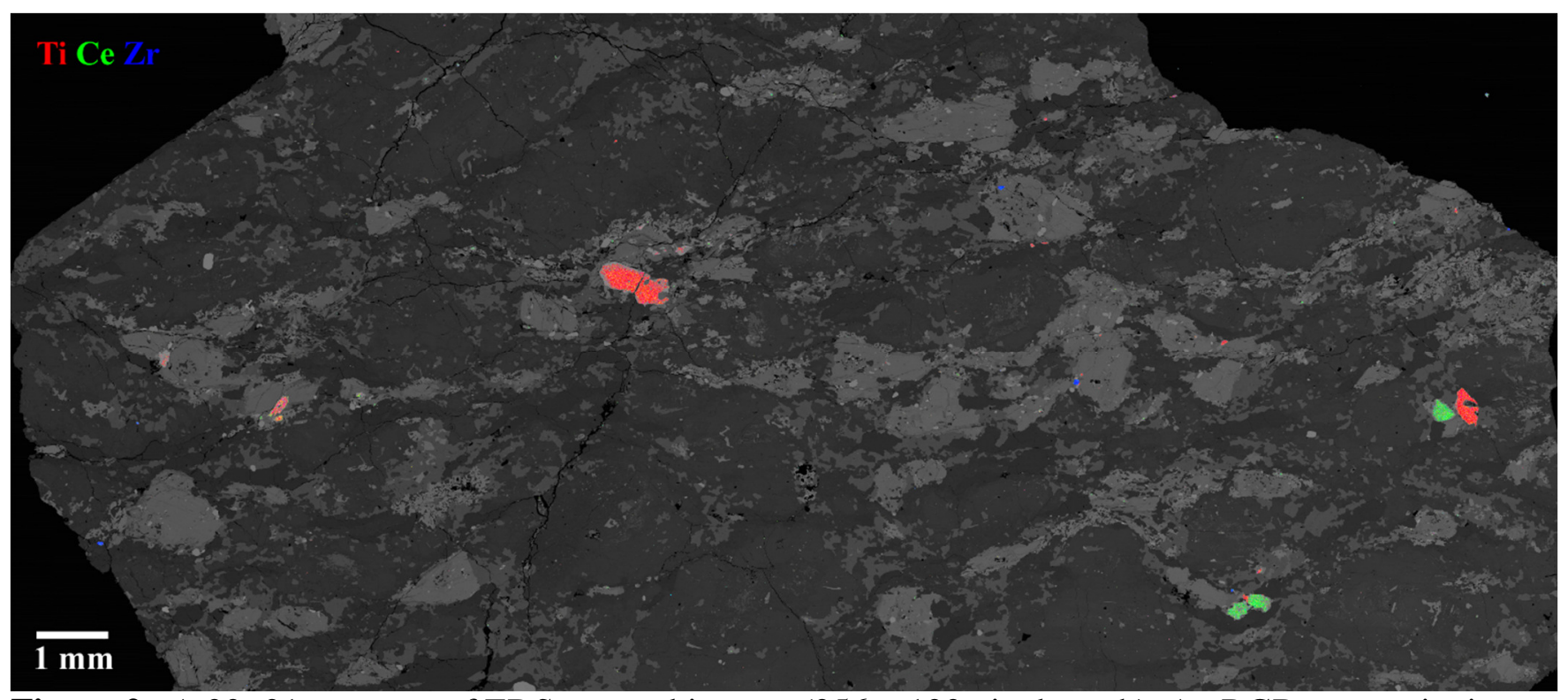

Figure 2. A $33 \times 21$ montage of EDS spectral images $(256 \times 192$ pixels each). An RGB composite image of net counts (background removed, peaks deconvolved) elemental X-ray maps of Ti (red), Ce (green), and $\mathrm{Zr}$ (blue) is displayed over the corresponding BSE image. 